\title{
Model-guided Lagrangian observation and simulation of mountain polar stratospheric clouds
}

\author{
M. Wirth, ${ }^{1}$ A. Tsias, ${ }^{2}$ A. Dörnbrack, ${ }^{3}$ V. Weiß, ${ }^{1}$ K. S. Carslaw, ${ }^{2,4}$ M. Leutbecher, ${ }^{3}$ \\ W. Renger, ${ }^{1} \mathrm{H}$. Volkert, ${ }^{3}$ and T. Peter ${ }^{2,5}$
}

\begin{abstract}
Gravity-wave-induced polar stratospheric clouds (PSCs) were observed over the Scandinavian mountains by airborne lidar on January 9, 1997. Guided by the forecasts of a mesoscale dynamical model, a flight path was chosen to lead through the coldest predicted region parallel to the wind at the expected PSC level $(23-26 \mathrm{~km})$. Because of the nearly stationary nature of the wave-induced PSC the individual filaments visible in the backscatter data of the clouds can be interpreted as air parcel trajectories. Assuming dry adiabatic behavior and fixing the absolute temperature to the ice frost point in the ice part of the cloud enables detailed microphysical simulations of the whole life cycle of the cloud particles. Optical calculations are used to adjust open parameters in the microphysical model by optimizing the agreement with the multichannel lidar data. This case is compared with former work from the Arctic winter 1994/1995. The influence of the stratospheric $\mathrm{H}_{2} \mathrm{SO}_{4}$ content and the cooling rate on the type of cloud particles (liquid ternary solution droplets or solid nitric acid hydrates) released from the ice part of the cloud is evaluated.
\end{abstract}

\section{Introduction}

It is now well recognized that heterogeneous chemical reactions on polar stratospheric cloud (PSC) particles play a key role in the activation of catalytically active halogen radicals from relatively inert reservoir compounds [World Meteorological Organization (WMO), 1992, 1994]. It is this activation process that leads to the photo catalytic reaction cycles that produce the so-called ozone hole. At present, we have a fairly detailed qualitative picture of these processes, but an accurate quantitative understanding is still lacking. Especially our ability to predict the number density, surface area, composition, and phase of particles, which will be generated in an atmosphere of given composition and temperature history, is still insufficient [Tolbert, 1994; Peter, 1997; Koop et al., 1997].

Carslaw et al. [1998a,b] showed that in the Arctic, mesoscale temperature fluctuations induced by mountain waves

\footnotetext{
${ }^{1}$ Arbeitsgruppe Lidar, DLR-Oberpfaffenhofen, Germany.

${ }^{2}$ Max-Planck-Institut für Chemie, Mainz, Germany.

${ }^{3}$ Institut für Physik der Atmosphäre, DLR-Oberpfaffenhofen, Germany. dom.

${ }^{4}$ Now at Environment Centre, University of Leeds, United King-

${ }^{5}$ Now at Institute of Atmospheric Sciences, ETH, Zuerich, Switzerland.
}

Copyright 1999 by the American Geophysical Union.

Paper number 1998JD100095.

0148-0227/99/1998JD100095\$09.00 are one source of PSC formation not covered by synoptic scale analyses, as for example that carried out by Pawson et al. [1995]. These wave-induced PSCs provide an outstanding opportunity to study the microphysics of particles by airborne (lidar) measurements. The small scale and quasi stationarity of these clouds allow a quasi-Lagrangian measurement strategy to be applied which covers the whole life cycle of a PSC particle. Besides the microphysical aspects these mesoscale PSCs cause air to be processed by heterogencous chemical reactions to an extent that may have a significant impact on global ozone chemistry. It should be noted that such wave-induced PSCs have been frequently documented for a long time [Störmer, 1929] and were previously termed mother-of-pearl or nacreous clouds.

Carslaw et al. [1998b] applied the quasi-Lagrangian approach to older observational material from the Second European Stratospheric Arctic amd Mid-latitude Experiment (SESAME, 1995) where only small portions of the flight track were accidentally aligned along the wind direction at PSC level. During the Airborne Polar Experiment (APE) we were able to plan the flight tracks of the aircraft more accurately in advance by employing a forecast system based on a mesoscale model driven by global forecasts [Dörnbrack et al., 1998].

This study focuses on a PSC encounter over the Scandinavian mountains on January 9, 1997. We show results of airborne lidar measurements, the mesoscale meteorological simulations that guided them, and compare the results of a microphysical and optical modeling against the lidar data. Section 4.3 will also compare these results with those of the first study from the winter of 1995 [Carslaw et al., 1998b]. 


\section{Mesoscale Dynamics}

In recent years it became increasingly recognized that mesoscale features (horizontal scales between 2 and $2000 \mathrm{~km}$ ) have to be taken into account also at stratospheric levels [e.g., Appenzeller and Davies, 1992]. Mesoscale wave motions in the stratosphere, which were excited by airflow over an underlying mountain range, have been documented [e.g., by Shutts et al., 1988].

\subsection{Modeling System}

In the work of Leutbecher and Volkert [1996] the fifth generation Pennsylvania State University / National Center for Atmospheric Research mesoscale model (MM5) [see Dudhia, 1993; Grell et al., 1994] was used for the simulation of wave propagation up to $30 \mathrm{~km}$. Using the same modeling system, Dörnbrack et al. [1998] presented mesoscale forecast and hindcast runs for the wave event on January 9, 1997. For the more detailed analyses presented here, we run a simulation with a twofold grid refinement (nested domains; see Figure 1) to better resolve the fine scale features of the Scandinavian mountain range. The coarsest computational domain with $36 \mathrm{~km}$ horizontal resolution is the same as described by Dörnbrack et al. [1998] (their Figure 1). The two nested domains provide high-resolution data inside the operational area of APE. The boundary and initial conditions for the model run were prescribed by global analyses of the European Centre for Medium-Range Weather Forecasts (ECMWF) with a spatial resolution of $2.5^{\circ}$ in latitude and longitude and on 14 standard pressure levels from the surface to $10 \mathrm{hPa}$. The model setup of the numerical simulations is the same as in the work of Dörnbrack et al. [1998].

\subsection{Wave Event, January 9, 1997}

2.2.1. Synoptic situation. Stratospheric analyses from ECMWF data around January 9, 1997, suggest a minimum temperature of $188-193 \mathrm{~K}$ in the center of the polar vortex near the pole. The edge of the polar vortex was located above the mountains of northern Scandinavia and the synoptic temperatures ranged from 196 to $198 \mathrm{~K}$ at the $550 \mathrm{~K}$ isentropic surface $(\approx 23 \mathrm{~km})$ (Figure 2 ).

Thus temperatures upstream of the Scandinavian mountains at PSC levels were about $3-4 \mathrm{~K}$ too high for the presence of PSC type I. In the troposphere a fast moving frontal system was approaching Scandinavia from northwest. Early in the morning of January 9 , the cold front increased the near-surface wind speed up to $20 \mathrm{~m} / \mathrm{s}$ and changed the wind direction below $5 \mathrm{~km}$ toward northwest leading to conditions with almost no directional shear with height. Simultaneously, a jet stream at $10 \mathrm{~km}$ reached maximum wind speeds of more than $50 \mathrm{~m} / \mathrm{s}$. The air masses at the mountain crest levels became more stable as a reaction to the front passage in the first half of January 9, 1997.

The Froude number $\mathcal{F}=U /\left(N h_{0}\right)$ characterizes the flow regime, where $U$ and $N$ are the mean wind speed and the buoyancy frequency of the low-level flow upstream of the orography, and $h_{0}$ is its crest height. For $\mathcal{F}>1$, the oncoming flow has enough kinetic energy to pass over
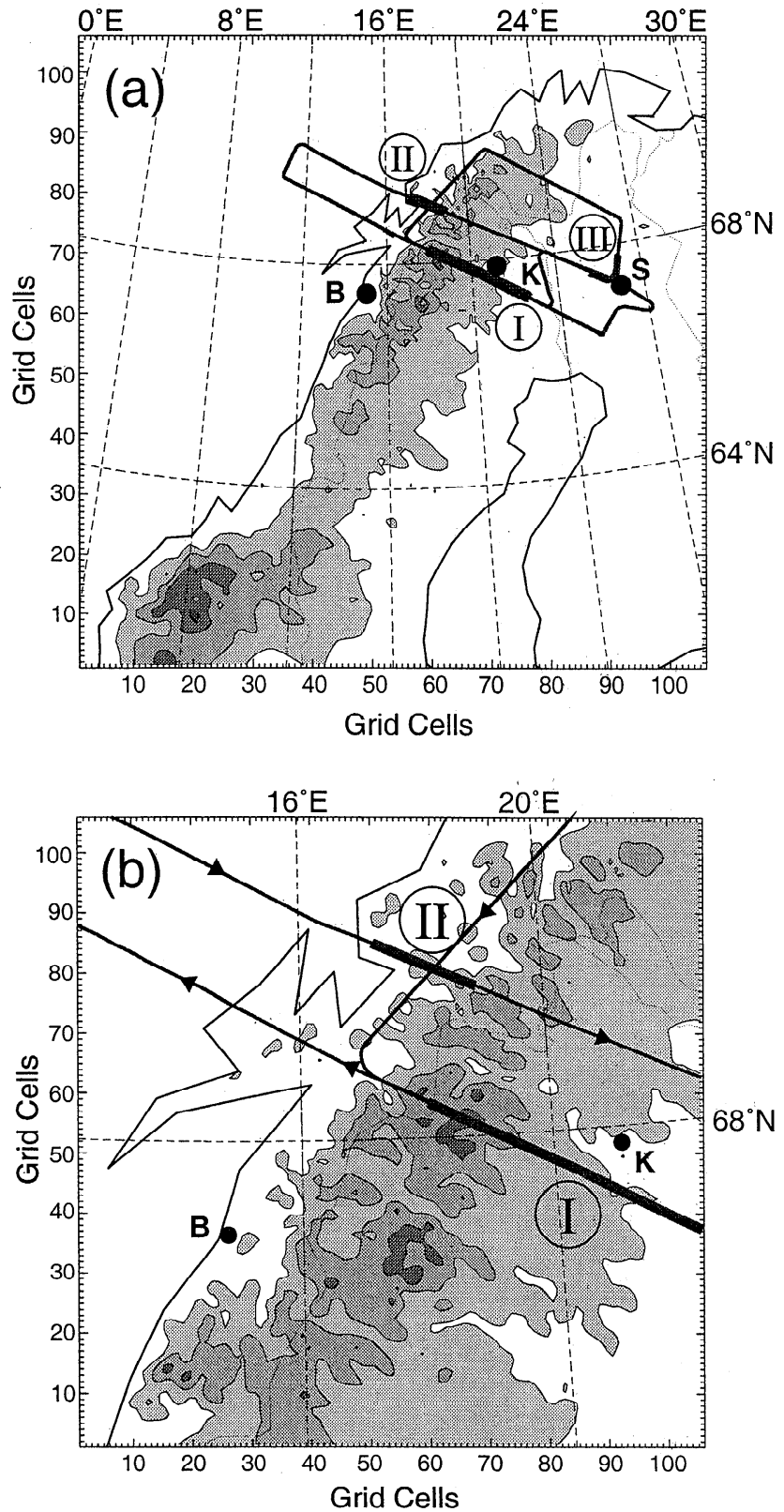

Figure 1. Subdomains of the mesoscale model: (a) $12 \mathrm{~km}$ resolution, $106 \times 106$ meshes and $1272 \mathrm{~km}$ subdomain length, and (b) $4 \mathrm{~km}$ resolution, $106 \times 106$ meshes and $424 \mathrm{~km}$ subdomain length. The model orography is given in increments of $400 \mathrm{~m}$. The flight path of the DLR-Falcon aircraft on January 9, 1997, is thickened in the parts where PSC clouds were observed by the lidar (numbers I to III). Locations mentioned in the text are marked by B, Bod $\varnothing ; \mathrm{K}$, Kiruna; S, Sodankylä.

the mountain. For $\mathcal{F}<1$, the flow is partially or totally blocked and is diverted around the mountains [Pierrehumbert and Wyman, 1985; Baines, 1995]. The mountain range represented by the model orography has a maximum elevation of about $1500 \mathrm{~m}$. The simulated Froude number $\mathcal{F}=20 \mathrm{~ms}^{-1} /\left(0.014 \mathrm{~s}^{-1} h_{0}\right) \approx 1430 \mathrm{~m} / h_{0}$ is close to 1 . Therefore most of the air is passing over the mountain ridge.

Altogether, the high near-surface winds, the uniformity of wind direction with height, the thermally stabilized air 

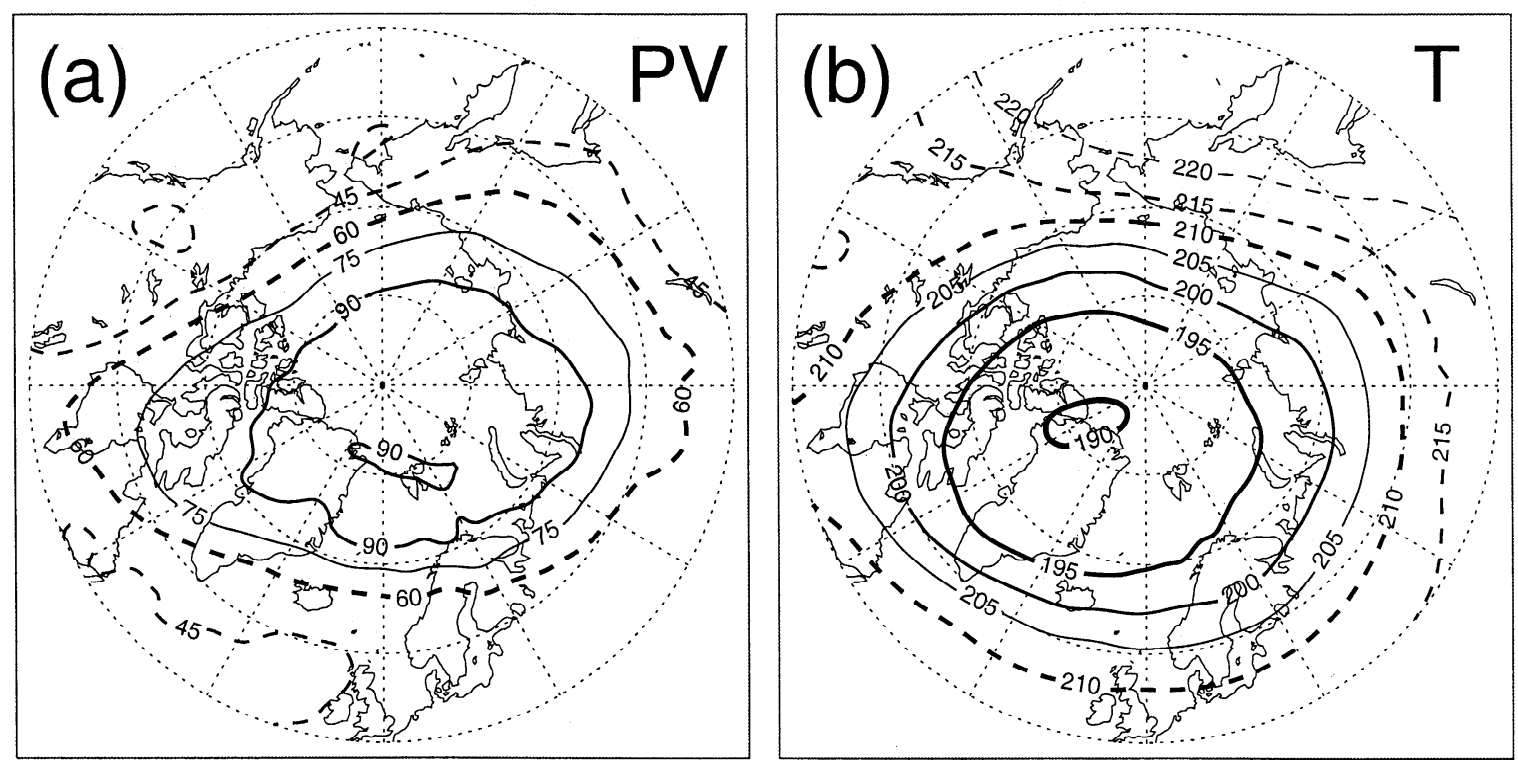

Figure 2. (a) Potential vorticity in units of $\mu \mathrm{K} \mathrm{m}^{2} \mathrm{~kg}^{-1} \mathrm{~s}^{-1}$ and (b) temperature in Kelvins on the $550 \mathrm{~K}$ isentropic surface for January 9, 1997. Data were taken from the European Centre for Medium-Range Weather Forecasts (ECMWF) 1200 UTC analyses. On this day, high PV is collocated with low temperature. Northern Scandinavia was located at the vortex boundary.

masses at mountain crest level, and the strong jet stream at the edge of the polar vortex favored the excitation and vertical propagation of mountain waves into the stratosphere.

2.2.2. Temperature at PSC levels. The numerical simulation of the flow allows to quantify the mesoscale temperature anomalies induced by the mountain waves in the stratosphere. Plate 1 shows the horizontal temperature structure at the $600 \mathrm{~K}$ isentropic surface for both nested domains of the mesoscale model, and Figure 3 depicts the vertical section above the first flight leg of the Falcon aircraft at about 1630 UTC.

A mesoscale temperature anomaly is visible above the mountain ridge as an isolated, elongated, and coherent cold spot of about $125 \mathrm{~km}$ east-west extension with minimum temperatures of less than $185 \mathrm{~K}$ (Plate 1). The north-south extension of this temperature minimum amounts to approximately $450 \mathrm{~km}$. The maximum temperature decrease of $13 \mathrm{~K}$ (related to upstream temperatures at the same isentropic surface) results from the adiabatic expansion of the ascending air. In the coldest region the $600 \mathrm{~K}$ isentropic surface is lifted by nearly $1300 \mathrm{~m}$. Although the steepening of the isentropic surfaces is rather large, the wave amplitude is not sufficient to result in overturning. This indicates that there was no wave breaking on the scales resolved, neither in the stratosphere nor in the troposphere.

Farther to the east a warm region is followed by a second cold region which is located $300 \mathrm{~km}$ downstream and west of the Kola peninsula. This second cooling is caused by slant propagation of internal gravity waves under the action of the Coriolis force [Queney, 1948].

In the vertical section the region of lowest temperatures $(T<185 \mathrm{~K})$ is located between $18^{\circ} \mathrm{E}$ and $19^{\circ} \mathrm{E}$, directly above the highest peaks of the orography in the mesoscale model (see Figure $3 \mathrm{~b}$ ). Its horizontal widths of $20 \mathrm{~km}$ is well correlated with the typical width of the peaks of model orography underneath. The temperature gradient at an altitude of $25 \mathrm{~km}$ between the temperature minimum (at $\left.18.7^{\circ} \mathrm{E}\right)$ and the maximum (at $21^{\circ} \mathrm{E}$ ) amounts to $20 \mathrm{~K}$ within $150 \mathrm{~km}$.

2.2.3. Radiosondes: Bodø, Kiruna, and Sodankylä. To estimate the accuracy of the mesoscale model simulations, we compare the calculated stratospheric temperature structure with soundings of radiosondes launched on the afternoon of January 9 (Figure 4).

The Bod $\varnothing$ sonde sampled the southern end of the cold spot above the mountain range at an altitude of about $22 \mathrm{~km}$, and the Sodankylä sounding passed the easterly temperature minimum downstream at heights between 23 and $25 \mathrm{~km}$. Both cold spots are reproduced by the MM5 soundings which follow the balloon trajectories. Unfortunately, the radiosondes give no information for regions where PSCs were actually observed by the lidar as the Bodø sonde missed this region by passing south and the other sondes are launched downstream of the westerly cold spot. Thus the only direct indicator of a stratospheric temperature minimum above the mountain ridge is located south of the Kebnekajse massif with $T=192.9 \mathrm{~K}$ at $25.5 \mathrm{~km}$ from the Bod $\varnothing$ sonde at 1200 UTC. The Bodø sonde launched 12 hours later measured a minimum temperature of $189.9 \mathrm{~K}$ at $22 \mathrm{~km}$. The MM5 temperatures following the Bodø and the Sodankylä sondes are about $3 \mathrm{~K}$ too warm when compared with the measurements at PSC levels $(23-26 \mathrm{~km})$.

Thus we conclude that the minimum temperatures in the coldest regions derived from the mesoscale model simulations are in the range of $184 \pm 3 \mathrm{~K}$. Differences between the simulated and the real temperature fields may arise from a poor vertical resolution of the lateral boundary conditions 


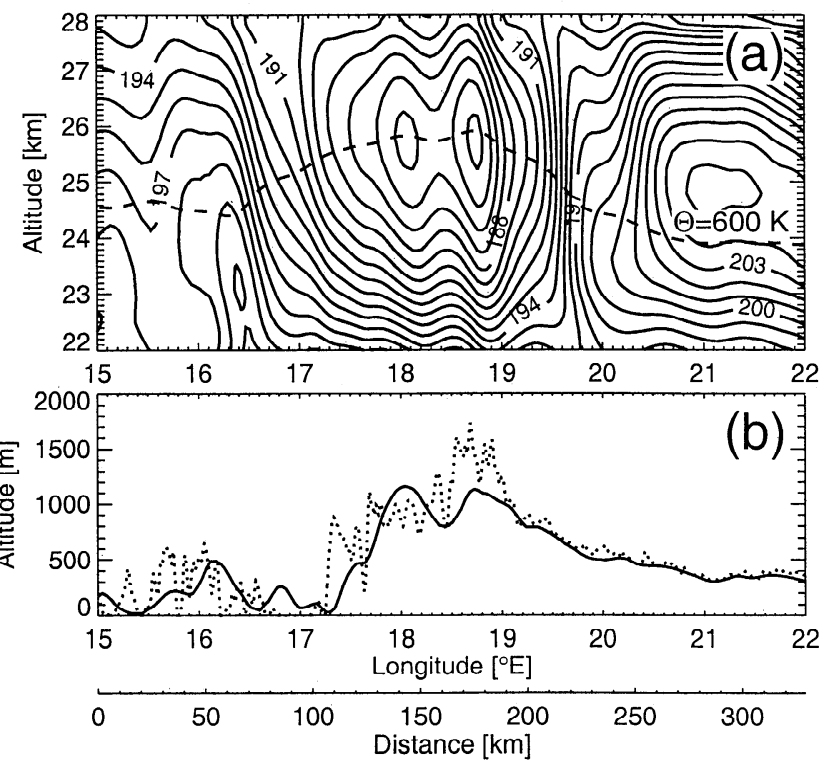

Figure 3. (a) Vertical section of temperature in Kelvins along the flight leg shown in Plate 1b. Data are taken from the innermost nest at 1630UTC. The dashed line shows the altitude of the $\Theta=600 \mathrm{~K}$ isentropic surface (compare Plate 1). (b) Terrain heights along the baseline of the flight legs: MM5 orography in the finest domain (solid black), and $20^{\prime \prime}$ orographic data set orography provided by the Geophysical Data Center in Boulder for the first east to west flight leg of the Falcon (dotted).

in the stratosphere and from the presence of shorter waves not resolved by the model. The lidar measurements shown later suggest an additional temperature fluctuation of about $\pm 2 \mathrm{~K}$ at a wavelength smaller than $20 \mathrm{~km}$ which cannot be resolved by the simulation (compare the discussion in section 4.2).

\section{Quasi-Lagrangian Lidar Measurements}

\subsection{Lidar System}

A multiwavelength aerosol and ozone lidar called OLEX (Ozone Lidar Experiment) was flown onboard the Falcon F-20 aircraft. A precursor of this lidar system had been operated during several Arctic campaigns onboard the Transall aircraft, [see Wirth and Renger, 1996; Carslaw et al., 1998b]. For the detection of PSCs the OLEX supports three aerosol detection channels; two different polarizations at $532 \mathrm{~nm}$ laser wavelength and one UV channel at $355 \mathrm{~nm}$. Table 1 shows the relevant system parameters. The lidar data are presented as total backscatter ratio $(S)$ and aerosol depolarization $\delta_{\mathrm{A}}$ at $532 \mathrm{~nm}$ wavelength and the color ratio. In terms of the backscatter coefficient $\beta$, these optical properties are defined as

$$
\begin{array}{r}
S=\left(\beta_{\text {aerosol }}+\beta_{\text {air }}\right) / \beta_{\text {air }} \\
\text { color ratio }=\beta_{\text {aerosol }}^{355 \mathrm{~nm}} / \beta_{\text {aerosol }}^{532 \mathrm{~nm}} \\
\delta_{\mathrm{A}}=\beta_{\text {aerosol, } \perp \perp} / \beta_{\text {aerosol, } \|}
\end{array}
$$

The backscatter coefficient of clear air $\beta_{\text {air }}$ is proportional to the molecular number density of the air and was calculated from the pressure and temperature data of the mesoscale model. In the region of strong wave excitation there is still about $3 \mathrm{~K}$ difference between the model results and the temperature estimated from the frost point (compare section 4.2) or taken from radiosondes (see section 2.2.3). A first-order estimation shows that this results in an error of about $\pm 1 \%$ in $\beta_{\text {air }}$ which can be neglected for our purposes.

To preserve the high resolution of the lidar data, we did not correct for aerosol extinction. However, from the shadow cast by the thickest ice cloud in Plate 2 on the clear air above, an optical thickness of about 0.1 can be estimated. Thus the maximum error in the backscatter ratio due to aerosol
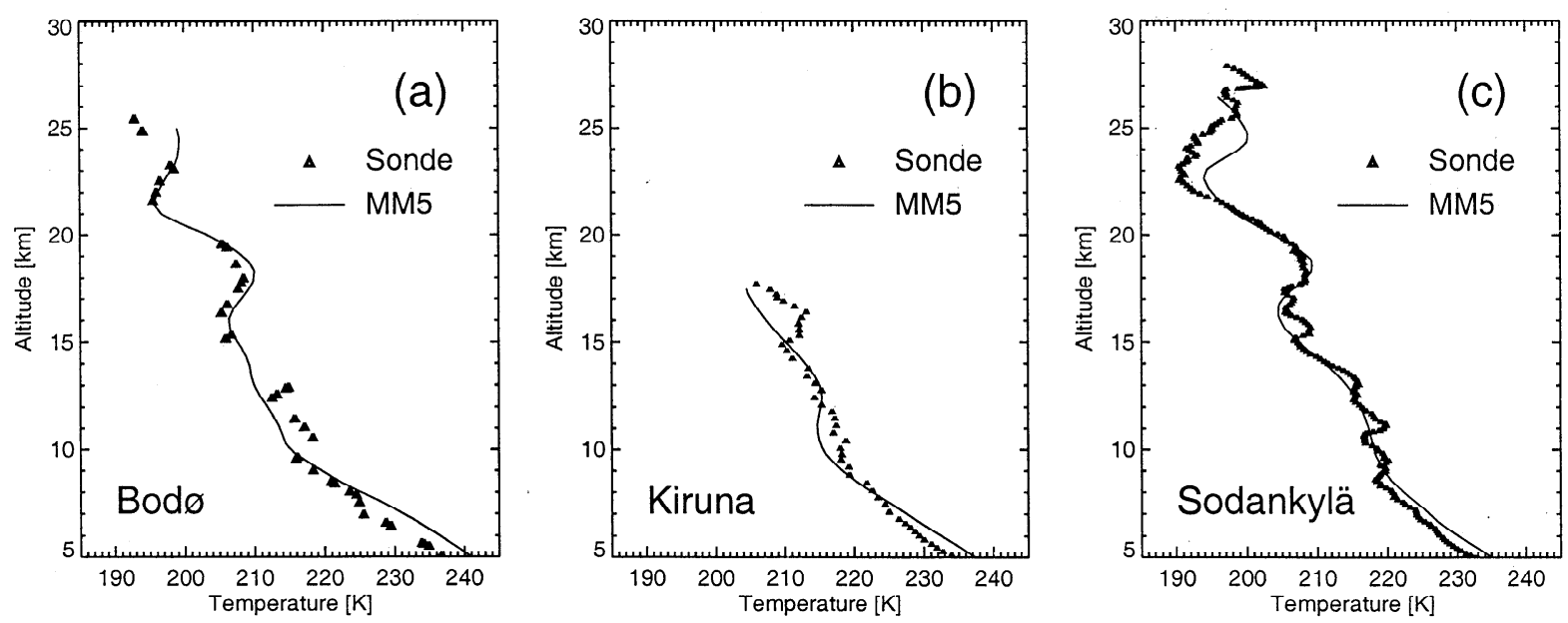

Figure 4. Vertical temperature soundings by radiosondes (marked by symbols) and MM5 temperature profiles following the balloon trajectory on the afternoon of January 9, 1997. (a) Sounding Bod $\varnothing$ at 1200 UTC. Data are on standard pressure levels and at significant levels. The trajectory of the balloon is calculated by assuming a constant ascent rate of $4.5 \mathrm{~m} / \mathrm{s}$. (b) Sounding Kiruna at 1400 UTC. Data (including ascent rate and position) are available every $10 \mathrm{~s}$ up to $1800 \mathrm{~m}$, every $30 \mathrm{~s}$ up to $6800 \mathrm{~m}$ and every minute up to the bursting level. (c) Sounding Sodankylä at 1800 UTC. Data (including ascent rate and position) are available every $2 \mathrm{~s}$ for the whole ascent. 


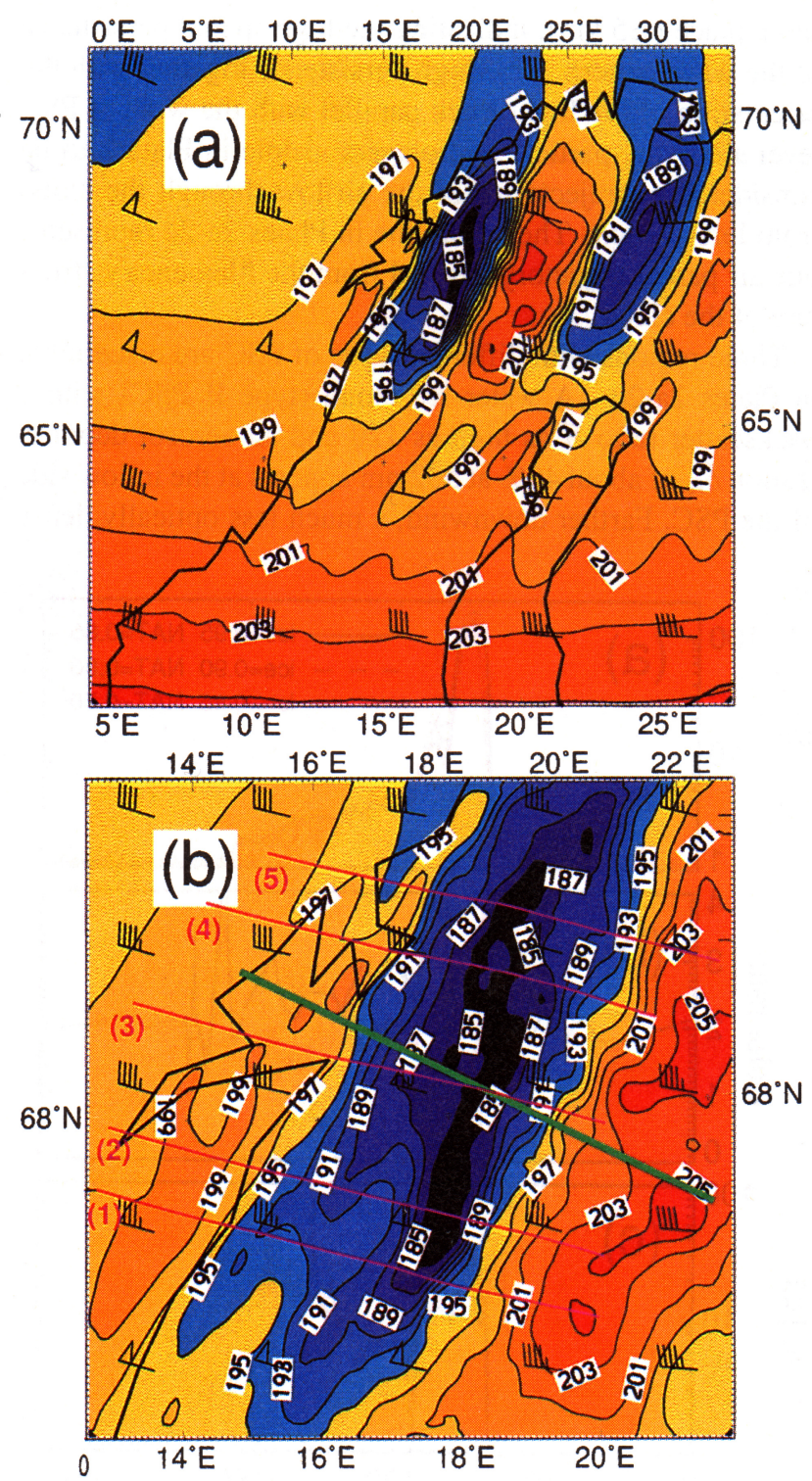

Plate 1. (a) Horizontal section of temperature in Kelvins at the isentropic surfaces $600 \mathrm{~K}(\approx 25 \mathrm{~km})$ for the medium resolution nest. The wind field is indicated by flags (short barb, $5 \mathrm{~m} / \mathrm{s}$; long barb, $10 \mathrm{~m} / \mathrm{s}$; triangle, $50 \mathrm{~m} / \mathrm{s}$ ). (b) Same as Plate la for the high-resolution nest. The thick green line depicts the first east to west leg (1630 UTC) of the flight path (compare Figure 3 for a vertical section along this line). The pink lines indicate the tracks of the trajectories shown in Figure 6.

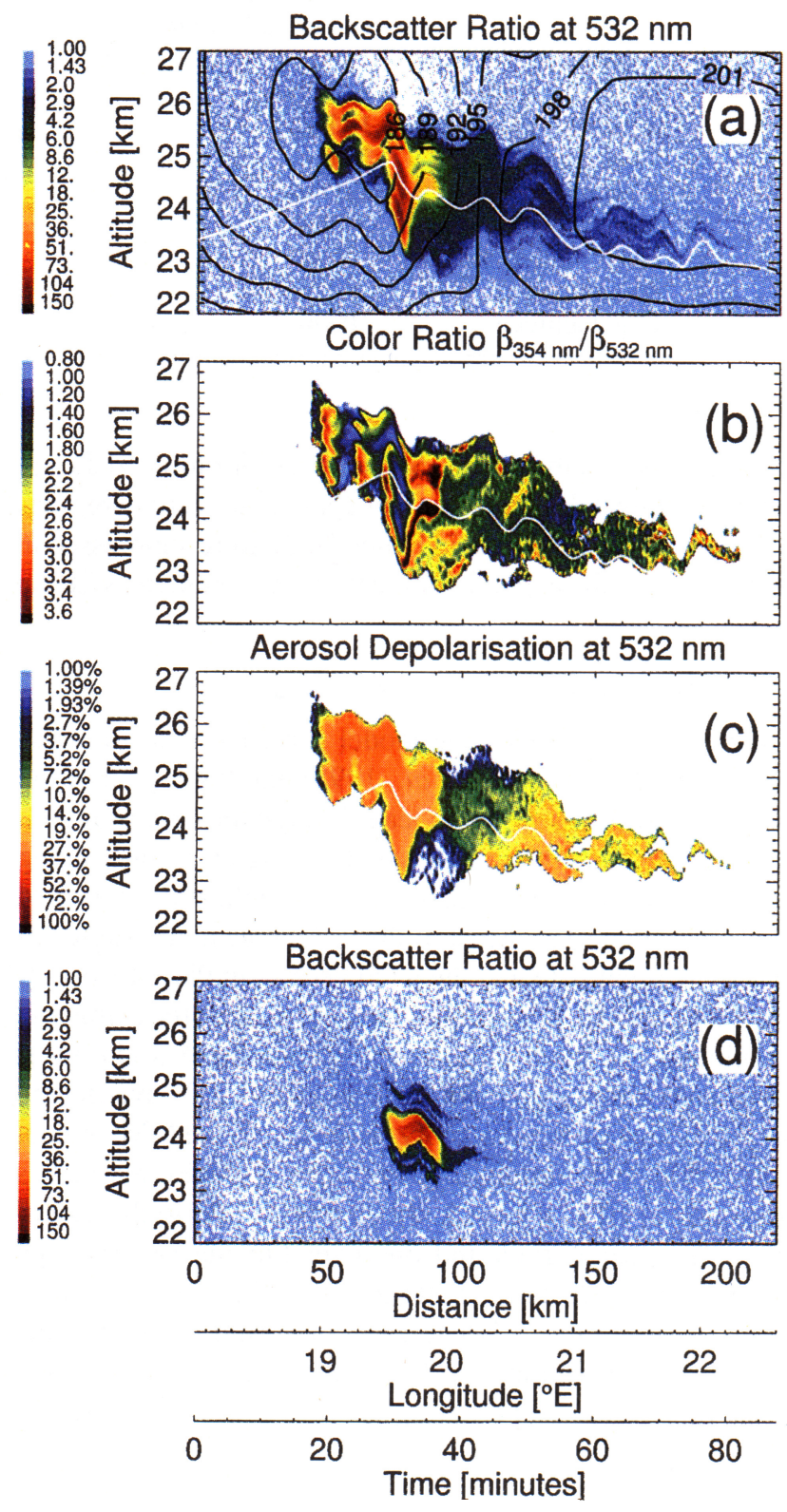

Plate 2. Lidar measurements of January 9, 1997, along flight leg I at 1615 UTC (a-c) and for the second pass at 1900 UTC at the same location (d): (a) backscatter ratio $S$ at $532 \mathrm{~nm}$ laser wavelength; (b) color ratio $\left(\beta_{\text {aerosol }}^{355 \mathrm{~nm}} / \beta_{\text {aerosol }}^{532 \mathrm{~nm}}\right)$; (c) aerosol depolarization ratio $\left(\delta_{\mathrm{A}}\right)$ at $532 \mathrm{~nm}$ laser wavelength; (d) backscatter ratio 2:45 hours later; The time axis refers to an air parcel flowing through the cloud with a wind speed of $150 \mathrm{~km} / \mathrm{h}$, as determined by the ECMWF analyses. The temperature contours in Plate $2 \mathrm{a}$ are from a MM5 model run with $4 \mathrm{~km}$ horizontal resolution. The white line in Plates $2 \mathrm{a}-2 \mathrm{c}$ depicts the assumed trajectory (see text). 
Table 1. Lidar System Parameters

\begin{tabular}{ll}
\hline Parameter & Typical Value \\
\hline Pulse energy & $300 \mathrm{~mJ}$ at $1064 \mathrm{~nm}$ \\
& $120 \mathrm{~mJ}$ at $532 \mathrm{~nm}$ \\
& $180 \mathrm{~mJ}$ at $355 \mathrm{~nm}$ \\
Pulse length & $1.8 \mathrm{~m}$ \\
Beam divergence & $0.5 \mathrm{mrad}$ \\
Repetition rate & $10 \mathrm{~Hz}$ \\
Telescope diameter & $35 \mathrm{~cm}$ \\
Field of view & $1.0 \mathrm{mrad}$ \\
Vertical resolution & $30 \mathrm{~m}$ \\
Horizontal resolution & $100 \mathrm{~m}$ \\
Aircraft speed & $200 \mathrm{~m} / \mathrm{s}$ \\
Flight altitude & $10 \mathrm{~km}$ \\
\hline
\end{tabular}

extinction is less than $10 \%$. Since the color ratio and $\delta_{\mathrm{A}}$ are quotients with a denominator that approaches zero in regions with low aerosol, the data in Plates $2 \mathrm{~b} / 2 \mathrm{c}$ were suppressed in regions where the aerosol backscatter was less then twice the detection limit.

\subsection{Lidar Results}

Guided by the mesoscale model simulations in forecasting mode [cf. Dörnbrack et al., 1998], the flight plan of the Falcon aircraft was set up to lead through the coldest region in several legs aligned in a quasi-Lagrangian manner along the (forecasts) of the stratospheric wind field at the expected PSC level (Plate 1b). Most of the difference in the angle between the flight path and the wind vectors in Plate $1\left(\approx 12^{\circ}\right)$ can be attributed to deviations between the forecasting and the hindcasting mode (the latter is shown here). Lidar measurements were taken from 1550 to 1945 UTC covering the period of highest wave activity according to the MM5 model runs. PSCs were found in three different places, marked as sections I to III in Figure 1: the first two close to the mountain ridge near Kiruna and one over Finland at very high altitude (up to $29 \mathrm{~km}$ ). Plate 2 shows lidar cross sections of backscatter ratio $S$ (Plate 2a), color ratio (Plate 2b) and aerosol depolarization $\delta_{\mathrm{A}}$ (Plate 2c) for the cloud in section I at the time of the first crossing (1615 UTC). Plate $2 \mathrm{~d}$ shows
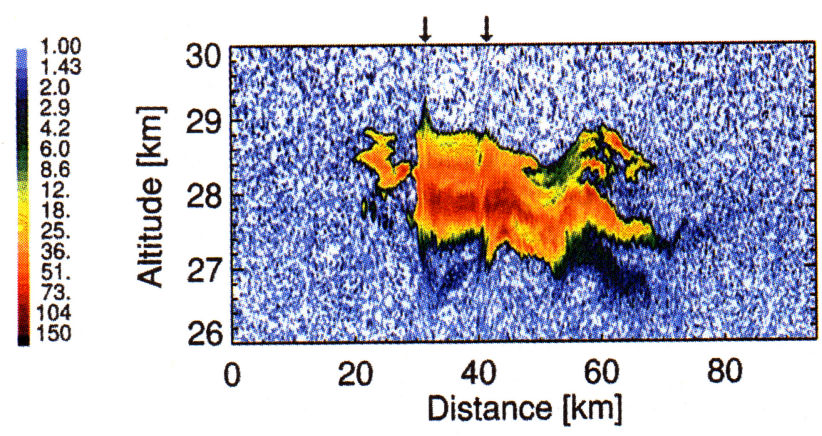

Plate 3. Lidar backscatter ratio $S$ for section III (compare Figure 1) of the flight track. Between the black arrow marks on top of the plot, the aircraft turned from southeast to northeast. In this region the lidar data are corrected for the light path elongation due to the nonzero roll angle of the aircraft. the backscatter ratio for the second underpass nearly 3 hours later. Since the mountain wave excitation was already decaying (a fact also reproduced by the mesoscale model), the region where PSC temperatures were reached was significantly smaller. However the core of the cloud stayed at the same place $( \pm 5 \mathrm{~km})$, indicating the high spatial persistence of the wave pattern. This high persistence together with the orientation of the flight track parallel with the wind at PSC level allow the particle microlayers visible in Plate 2 to be considered as trajectories of the airflow through the cloud from left to right. The white line in Plates $2 \mathrm{a}-2 \mathrm{c}$ represents one air parcel trajectory derived from the filamentary structure of the cloud.

Three regions with different types of PSC are discernible in Plates 2a-2c. A water ice cloud (type II PSC) with a backscatter ratio $S$ as high as 120 and an aerosol depolarization $\delta_{\mathrm{A}}$ of about $40 \%$ at $532 \mathrm{~nm}$ appears at the inflow side of the PSC. Farther downwind, a much less optically dense

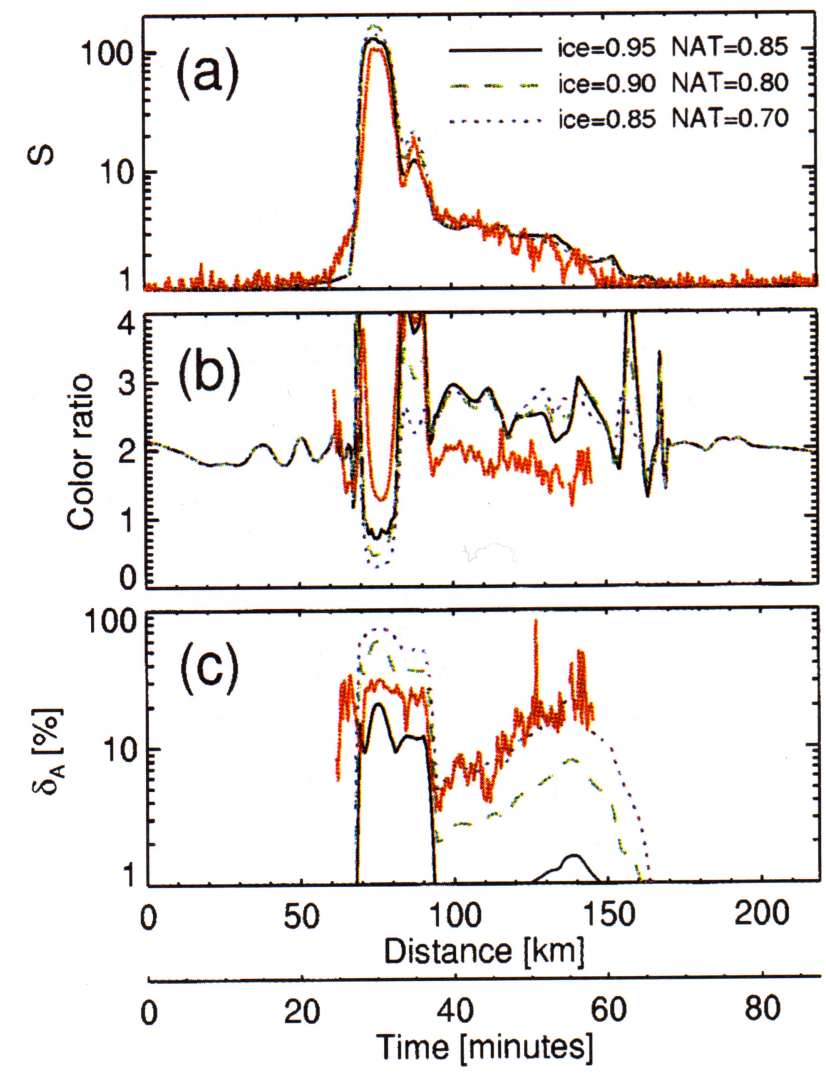

Plate 4. Comparison of lidar measurements with simulated scattering parameters along the white line shown in Plate 2: (a) observed backscatter ratio at $532 \mathrm{~nm}$ laser wavelength $S$ (red) and calculated $S$; (b) observed (red) and calculated color ratio $\left(\beta_{\text {aerosol }}^{355 \mathrm{~nm}} / \beta_{\text {aerosol }}^{532 \mathrm{~nm}}\right)$; (c) observed (red) and calculated aerosol depolarization ratio $\left(\delta_{\mathrm{A}}\right)$; the time axis refers to an air parcel flowing through the cloud with a wind speed of $150 \mathrm{~km} / \mathrm{h}$, as determined by the ECMWF analyses. Calculations are shown for prolate particles with three different aspect ratios: $0.85,0.9$, and 0.95 for ice and $0.7,0.8$, and 0.85 for NAT. A refractive index of 1.48 was used for NAT and 1.31 for ice. For liquid aerosols an index of refraction as a function of aerosol composition, temperature, and wavelength was used [Krieger et al., 1997]. 
filamentary structure trails the type II cloud. For the most part this cloud tail backscatters significantly in the perpendicular polarized channel, indicating the presence of solid (nonspherical) particles. In the lower-most part of the outflow region of the ice cloud (at $90 \mathrm{~km}$ distance and $23.5 \mathrm{~km}$ altitude in Plate 2) the particles show no significant aerosol depolarization but a medium backscatter ratio $(\approx 3)$, typical for the spherical particles of liquid ternary solutions.

The mesoscale dynamical simulation predicted also a cold spot over Finland and the Kola peninsula not directly correlated to any significant underlying orographical obstacle. Plate 3 shows the backscatter ratio $S$ measured by the OLEX lidar in section III of the flight track near Sodankylä. The peak values of 80 at $28 \mathrm{~km}$ altitude show the presence of water ice at synoptic temperatures about $14 \mathrm{~K}$ above the frost point. So even $300 \mathrm{~km}$ away from the mountains, waveinduced PSC can be formed. For the microphysical simulations we will concentrate on the cloud detected in section I the cloud in section II (not shown here) is much smaller, and section III is not aligned with the wind).

\section{Microphysical and Optical Simulations}

\subsection{Microphysical Model}

As in the work of Carslaw et al. [1998b], we have combined the lidar observations with microphysical and optical model simulations in an iterative process (Figure 5) to determine the size of the particles along an air parcel trajectory.

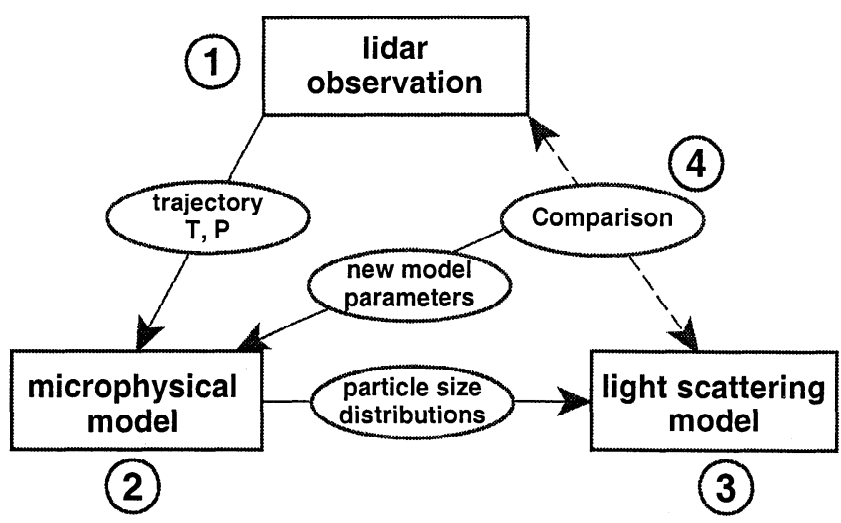

Figure 5. Schematic of the iterative process used to derive PSC characteristics using lidar observations, microphysical model simulations, and optical calculations. The lidar observations (number 1) were used to determine an air parcel trajectory along clear bands of aerosol. This yields the altitude and thus the ambient air pressure. With this information and an initial first guess of the particle number densities the microphysical model (number 2) was used to calculate the time development of liquid, ice, and NAT (or NAD) particle size distributions along the trajectory. The optical model (number 3 ) was then used to calculate a simulated lidar signal along the trajectory for the three optical properties total backscatter ratio, perpendicular backscatter ratio and color ratio. The simulated and observed lidar signals were then compared and the assumptions in the microphysical model refined. This process was then repeated in the order of 2 , $3,4,2$ until the agreement between the simulated and the observed lidar signals was optimal.
A T-matrix light-scattering algorithm [Mishchenko, 1991] suitable for nonspherical (axis symmetrical) particles was adopted. Size distributions calculated in the microphysical model were used as input to the optical code. The time development of particle composition and size was simulated using a fully kinetic microphysical Lagrangian box model. The model includes growth and evaporation of ternary $\mathrm{HNO}_{3} / \mathrm{H}_{2} \mathrm{SO}_{4} / \mathrm{H}_{2} \mathrm{O}$ (STS) droplets as well as the solid particles ice, nitric acid trihydrate (NAT), nitric acid dihydrate (NAD), and frozen sulfuric acid aerosols composed of sulfuric acid tetrahydrate (SAT). Equilibrium vapor pressures of ice and NAT particles were taken from Hanson and Mauersberger [1988] and the NAD vapor pressure according to Worsnop et al. [1993]. The liquid-only component of the model has been used in earlier simulations of nonequilibrium stratospheric liquid aerosol growth in mountain waves [Meilinger et al., 1995]. Tsias et al. [1997] examined the nucleation of NAD and NAT from nonequilibrium droplets, and later Carslaw et al. [1998b] investigated ice and NAT PSC growth in mountain waves over Scandinavia.

In detail the microphysical model proceeds as follows: When a droplet freezes, an initial ice crystal is assumed to form instantaneously from the water molecules available in the droplet, until equilibrium is reached between the remaining liquid and the ice. The ionic concentration of this remaining liquid is of course greater than in the droplet before freezing and can be calculated from thermodynamic equilibrium considerations. The ice crystal then continues to grow by gas phase vapor diffusion. The ice particle is assumed to be at equilibrium with the surrounding liquid layer during the ice particle growth process, which can be justified given the quasi-instantaneous equilibration between liquid and ice. The water vapor pressure over the ice/liquid particle is therefore that of pure ice, and it is this vapor pressure that is used in the ice growth equation. The calculated composition of the liquid layer in equilibrium with the ice particle determines the $\mathrm{HNO}_{3}$ vapor pressure, which is used to calculate $\mathrm{HNO}_{3}$ mass transfer between the gas phase and the liquid layer. This $\mathrm{HNO}_{3}$ transfer leads to a change in the mass of the liquid layer with time, but the ionic concentration at any moment is always determined by equilibrium between the liquid layer and the ice. Note that the concentration of $\mathrm{HNO}_{3}$ reached in the liquid layers surrounding ice particles is considerably lower than calculated to exist in nonequilibrium supercooled ternary solution (STS) droplets [Meilinger et al., 1996; Tsias et al., 1997] during similarly rapid cooling events. This is because of the requirement of liquid/ice equilibrium, which restricts the ionic concentrations to thermodynamic equilibrium values. The supersaturation with respect to NAT (or NAD) can be calculated in the normal way by examining the $\mathrm{HNO}_{3}$ and $\mathrm{H}_{2} \mathrm{O}$ partial pressures at any moment in time. Formation of NAT may occur either from within the liquid layer itself (freezing nucleation, possibly assisted by heterogeneous contact with the ice surface) or from $\mathrm{HNO}_{3}$ vapor deposition on any exposed ice surface (deposition nucleation). Our analysis of the ice cloud in Plate 2 suggests that deposition nucleation of NAT may be the favored process. 
To avoid numerical diffusion, particle growth was calculated using a Lagrangian scheme in radial space; that is, growth of individual size classes is followed rather than distributing mass changes between fixed radius bins. The size distributions of liquid and solid particles are each represented by 26 radius classes. Solidification of droplets, for example to ice or NAT, in a given radius class is assumed to be instantaneous and to occur only during a single time step of the model (that is, we do not consider an activation rate that would lead to the number of unfrozen particles in a given size class varying exponentially with time). However, the fraction of particles that solidify can be freely varied [see Tsias et al., 1997]. After solidification or partial solidification of particles in a given size class, the diffusive growth and evaporation of the liquid and solid particles is then separately followed. Such a purely Lagrangian treatment of particle growth would have limitations if ice particle nucleation were a slow process. However, as shown by Carslaw et al. [1998b], there is evidence that nucleation is rather rapid, as evidenced by the sharp changes in color ratio in their Plate 2. These peaks would have been considerably smoothed out had the nucleation of ice been a gradual process throughout the cloud. Such effects are also apparent in the lidar images shown here.

\subsection{Selection of Trajectory}

The most critical step for a successful microphysical simulation is the proper selection of the temperature history of the air parcel. In contrast to the case of January 15, 1995 [Carslaw et al., 1998a,b], it was not possible to read off the trajectory of an air parcel that covers the whole range of microphysical simulation from the lidar measurements alone. Since there is no sign of aerosol particles above the detection limit of the lidar on the inflow side of the cloud, we combined trajectories obtained from the mesoscale model runs with the finer scale signatures from the lidar (where they are available).

To get an overview of possible thermal histories, several trajectories were calculated within the mesoscale model runs. Figure 6 shows the temperature and the corresponding cooling/heating rates along trajectories that started at about $20 \mathrm{hPa}(24 \mathrm{~km})$ in the finest nested domain. The starting points are selected in such a way that the air parcels cross the western cold spot at about the time of the lidar measurement at 1630 UTC (see Plate 1b for the ground tracks).

Generally, the air parcels spend less than $5 \mathrm{~min}$ at temperatures below $T=185 \mathrm{~K}$, whereas on average, they stay about $20 \mathrm{~min}$ below $T=190 \mathrm{~K}$. Because of the perturbation of the flow by the mountain wave the mean horizontal wind speed of $42 \mathrm{~m} / \mathrm{s}(\approx 150 \mathrm{~km} / \mathrm{h})$ is modulated with lower values of $30-35 \mathrm{~m} / \mathrm{s}$ in regions of ascending motion and higher ones $(50-60 \mathrm{~m} / \mathrm{s})$ in regions of descent. Therefore the heating rates generally reach higher absolute values $(>100 \mathrm{~K} / \mathrm{h})$ than the cooling rates $(\approx 70 \mathrm{~K} / \mathrm{h})$. As the orography is better resolved than in the work of Dörnbrack et al. [1998], smaller-scale mountain waves are excited, which produce lower temperatures (minimum 183.6 K). However, as can be

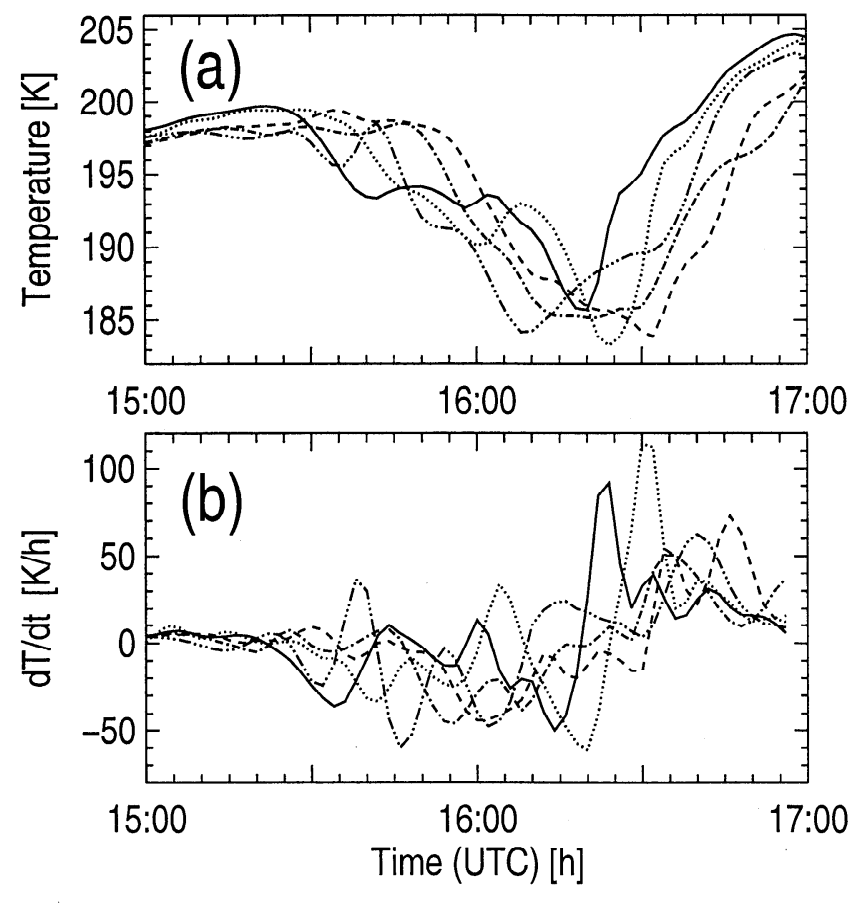

Figure 6. (a) Histories of temperature and (b) cooling/heating rates along individual trajectories of air parcels started in the innermost nest at 1500 UTC on January 9, 1997, at about $24 \mathrm{~km}$. The trajectories terminate at 1700 UTC. Line coding from south to north: solid line, 1; dotted, 2; dashed, 3; dashed-dotted, 4; dashed-triple dotted, 5 .

seen from the terrain heights along the baseline of the Falcon flight leg (Figure 3b), there is still a considerable difference in the top height and shape between the fine scale orography used by the mesoscale model and the best available digital data set (the real orography is even finer structured). So we expect that the simulated wave amplitudes would increase even further if the model resolution could be increased. This may improve the agreement between the dynamical calculations and the radiosonde and lidar observations.

Figure 7 shows the synthesized temperature history which was actually used for the microphysical simulations for the range of the lidar measurements in Plate 2 along with the result from the mesoscale model for trajectory 2 from Figure 6. This temperature history corresponds to the air parcel trajectory indicated by the white line in Plates $2 \mathrm{a}-2 \mathrm{c}$. Within the cloud the absolute temperature along the trajectory is determined by reference to the ice frost point level, which we fix as indicated in Plate 2a, midway between the peak in backscatter ratio and the point of ice disappearance [Carslaw et al., 1998b]. Temperatures at other points are then calculated by assuming dry adiabatic behavior. Outside of the PSC the temperature is smoothly adjusted to the mesoscale model results. Taking into account that the modeled temperatures seem to be about $3 \mathrm{~K}$ to warm, as compared to radio soundings (see section 2.2.3), and do not include small-scale waves $(\lambda<20 \mathrm{~km})$, there is a good agreement of the shape 

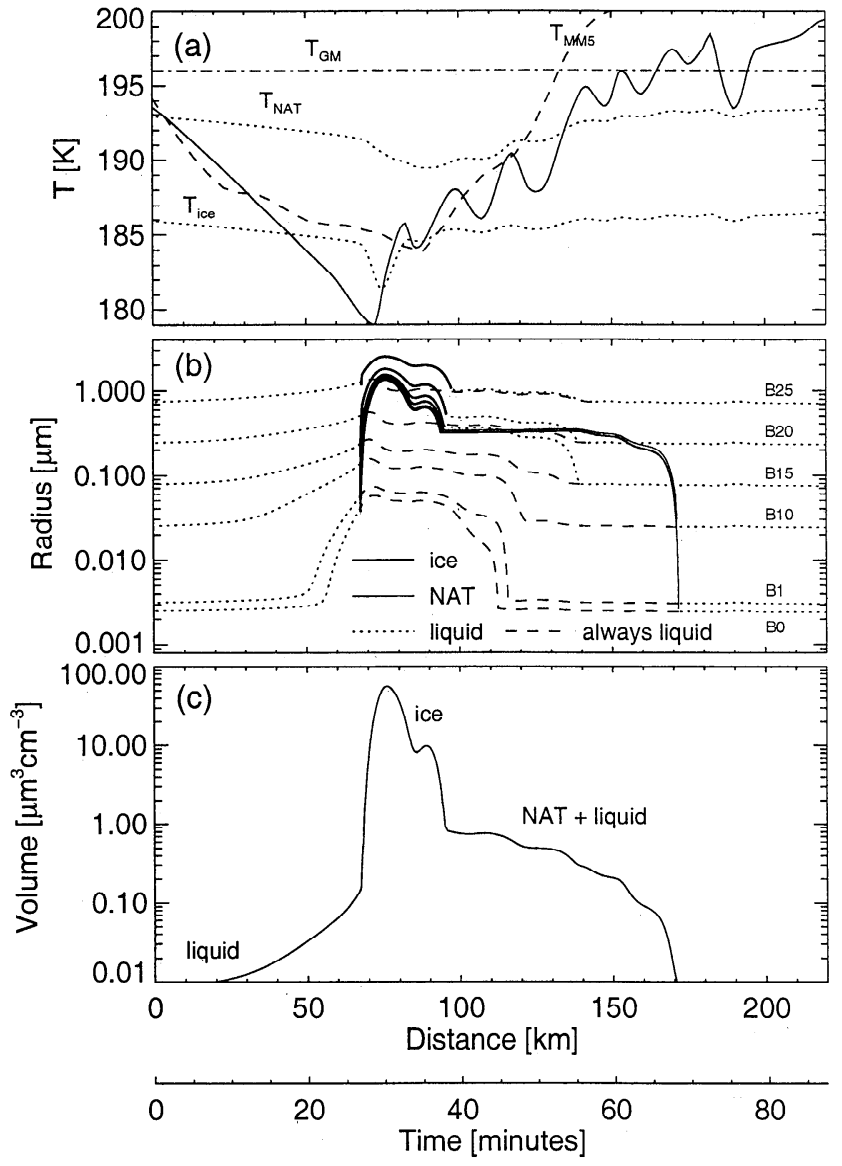

Figure 7. Microphysical box model simulation of the PSC event in Plate 2: (a) temperature; (b) particle radii (thin lines, NAT; thick line, ice; dashed lines, liquid); (c) particle volume. The initial aerosol size distribution of liquid particles was assumed to be lognormal and represented by 26 size bins $\left(B_{0}-B_{25}\right)$ with a total of 10 particles $\mathrm{cm}^{-3}$, an initial width of $\sigma=1.8$ and mode radius of $0.03 \mu \mathrm{m}$ at $230 \mathrm{~K}$ (equivalent to a total amount of $\mathrm{H}_{2} \mathrm{SO}_{4}$ in the air parcel of $0.05 \mathrm{ppbv}$ ). The air contains total mixing ratios of $5 \mathrm{ppmv} \mathrm{H}_{2} \mathrm{O}$ and 10 ppbv $\mathrm{HNO}_{3}$. For the simulation run shown here, virtually all droplets were assumed to freeze as ice $4.0 \mathrm{~K}$ below the ice frost point and were then allowed to grow as ice/liquid mixtures. For reference an "infinitesimal" fraction of $0.1 \%$ in all bins was kept in the liquid state. Nucleation of NAT was assumed to occur on ice particles belonging to bins 0 to 12 (60\% of the particles), leading to release of NAT upon ice evaporation. The remaining $40 \%$ of the ice particles were assumed to release liquid droplets after ice evaporation. These droplets need about 12 min to fall back to the size of liquid particles that never had been frozen, which shows up in a bifurcation of the dashed lines for the bins $B_{15}-B_{25}$ in Figure $7 \mathrm{~b}$. The ice and NAT equilibrium temperatures are plotted as dotted lines in Figure 7a.

and the heating/cooling rates in the region where the temperature history can be read off from the lidar data.

\subsection{Results of Microphysical Simulations}

4.3.1. Nucleation of ice. The apparent absence of any particles upwind of the ice cloud is in contrast to the cases studied by Carslaw et al. [1998b] where well developed liquid aerosol clouds were clearly visible. This difference probably arises due to the much more rapid cooling rate in the mountain wave in the case shown in Plate 2 than for the PSCs studied by Carslaw et al. [1998b]. As shown in Figure 6, simulations using the MM5 model indicate a peak cooling rate of up to $70 \mathrm{~K} \mathrm{~h}^{-1}$ upwind of the cloud, with the air cooling from a temperature of about $200 \mathrm{~K}$ to $184 \mathrm{~K}$ within $40 \mathrm{~min}$. Thus the background aerosols are cooled from well above liquid PSC conditions in a time that is insufficient to allow for any significant $\mathrm{HNO}_{3}$ uptake by the aerosols. This lack of aerosol growth is well reproduced in the microphysical simulation shown in Figure 7 and the corresponding optical calculations in Plate 4. In contrast, in the cases analyzed by Carslaw et al. [1998b], temperatures were below liquid PSC formation conditions for much longer ( $\approx 3$ hours) before ice formed.

One free parameter of the microphysical simulation is the fraction of particles on which ice (resp. NAT) nucleates. Optimum agreement between the observed and the calculated optical properties in the cloud was achieved by assuming that between $50 \%$ and $100 \%$ of the droplets upwind of the cloud nucleated to form ice and that the smallest $60 \%$ of the ice particles then served as sites for NAT nucleation (bins $0-12$ in the model). The essentially complete ice nucleation is the same as in the earlier mountain wave PSCs studied by Carslaw et al. [1998b]. Also, as in the earlier observations, it was necessary to apply a super cooling of $4 \mathrm{~K}$ below the ice frost point $\left(T_{\text {frost }}\right)$ in order to accurately reproduce the timing of initial ice formation.

4.3.2. Nucleation of hydrates. The greatest difference between these observations and those studied by Carslaw et al. [1998b] is that solid type I PSCs appear in close proximity to the ice cloud rather than appearing only $200 \mathrm{~km}$ downwind. This reflects the much higher number of hydrate particles nucleated, which in turn affects their size and the rate at which they grow by diffusive vapor transfer. Carslaw et al. [1998b] suggested that the low number density of NAT particles nucleated on ice in the case they studied (about $2 \%$ of all ice crystals) might be explained by the comparative rates of deposition (gas to solid) and freezing nucleation of NAT on the ice surfaces. We now reexamine this hypothesis in the light of these new observations.

Carslaw et al. [1998b] argued that NAT formation probably occurs via deposition nucleation on ice surfaces that become exposed to the gas phase, while NAT nucleation by freezing nucleation is too slow on those ice particles that remain enclosed within an unbroken liquid layer. Hence NAT nucleation occurs on those ice particles that grow from the smallest droplets, since the liquid layer preferentially breaks on these particles (see Figure 8). Given the broadly similar altitude, ice surface area, and temperatures in the two cases, it might be expected that hydrate formation would proceed with similar efficiency. According to the Carslaw et al. mechanism, the much higher NAT number densities in this case would therefore imply smaller amounts of liquid aerosol prior to ice formation and hence a larger number of ice particles that become exposed to the gas phase. There are two reasons that could explain the low levels of STS we 


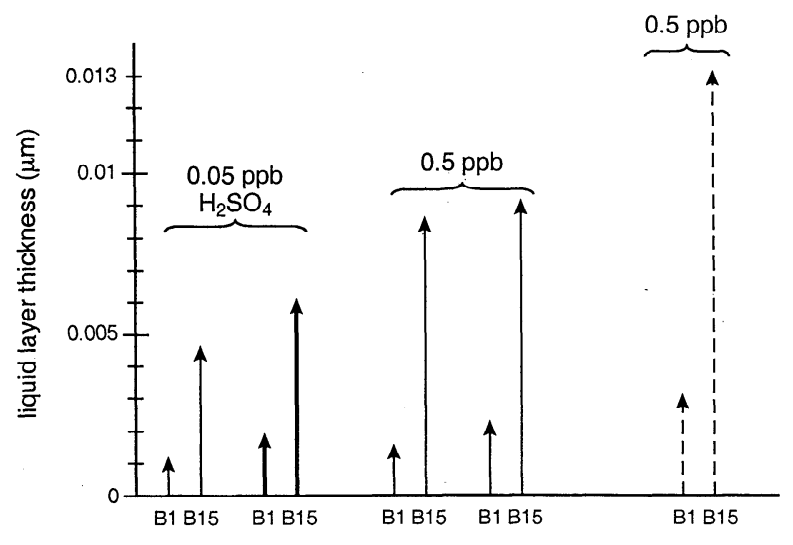

$\begin{array}{llllll}\text { cooling rate } \mathrm{K} \mathrm{h}^{-1} & 60 & 30 & 60 & 30 & \sim 15\end{array}$

Figure 8. Calculated minimum liquid layer thicknesses on spherical ice particles for two radius size classes $B_{1}$ and $B_{15}$ as shown in Figure 7. Thick solid lines, using the same conditions as in Plate 2 and Figure 7; thin solid lines, using the trajectory in Plate 2 with different initial cooling rates and different $\mathrm{H}_{2} \mathrm{SO}_{4}$ amounts; dashed lines, the case studied by Carslaw et al. [1998b]. In the process of particle growth this minimum liquid layer thickness is reached shortly before the ice particle radius reaches a maximum. We show results for bin $B_{15}$ since our simulations reveal that all droplets from the smaller size classes $B_{0}-B_{12}$ must be nucleated to NAT in order to explain the lidar observations.

observed on January 9. Firstly, as described above, the more rapid cooling rate on the upwind side of the wave in this case prevents significant aerosol growth due to $\mathrm{HNO}_{3}$ uptake. Secondly, the amount of $\mathrm{H}_{2} \mathrm{SO}_{4}$ aerosol in the stratosphere may have been still decaying after the Mount Pinatubo eruption in 1991 during winter 1994-1995 to winter 1996-1997 (S. Borrmann private communication, 1998). In combination, the lower amount of $\mathrm{H}_{2} \mathrm{SO}_{4}$ and/or the lack of growth due to $\mathrm{HNO}_{3}$ uptake mean that initial ice formation occurs in much smaller droplets than in the case studied by Carslaw et al. [1998b].

Figure 8 shows the results of a sensitivity calculation testing the effect of cooling rate and $\mathrm{H}_{2} \mathrm{SO}_{4}$ amount on the thickness of liquid layers on ice along the trajectory indicated in Plate 2. This comparison reveals that the liquid layers were as much as a factor of 2 thinner in this case (thick solid lines) compared with the Carslaw et al. [1998b] case (dashed lines). The calculations are consistent with NAT or NAD nucleation occurring on those ice particles whose liquid film thickness was less than about $6 \mathrm{~nm}$ (equivalent to $\sim 10-20$ monolayers of liquid coverage). These liquid layer thicknesses are considerably greater than a monolayer. However, we have not accounted for the tendency for liquids to form islands due to the surface tension. In addition, it is possible that the ice surface area may have been considerably greater than by assuming spherical ice particles [Keyser and Leu, 1993], thus making the liquid layers even thinner.

The calculations in Figure 8 reveal that a doubling of the cooling rate leads to at most a $25 \%$ decrease in minimum layer thickness, while a factor of 10 decrease in $\mathrm{H}_{2} \mathrm{SO}_{4}$ amount leads to a $50 \%$ decrease. Very large increases in $\mathrm{H}_{2} \mathrm{SO}_{4}$ amount, such as were present in the stratosphere in the first years after the eruption of Mount Pinatubo, might therefore be expected to reduce the number density of NAT particles produced in mountain wave clouds. For the difference between the measurements presented here and the Carslaw et al. [1998b] case (January 15, 1995) the amount of $\mathrm{H}_{2} \mathrm{SO}_{4}$ may have played a minor role, because there are midlatitude observations which show no further decay in aerosol amount after the year 1995 [Barnes and Hofmann, 1997], and in both cases, the PSCs were observed at an altitude (23-26 km) well above the upper boundary of the background aerosol layer within the Arctic vortex [Wirth and Renger, 1996]. For typical background $\mathrm{H}_{2} \mathrm{SO}_{4}$ amounts, as can be assumed here, the cooling rate has by far the largest influence on NAT nucleation. This suggests that NAT clouds forming downwind of mountains may have a wide range of particle number densities and therefore growth rates, depending on fine scale details of the flow dynamics.

We like to stress that the sensitivity calculations in Figure 8 show how cooling rate and $\mathrm{H}_{2} \mathrm{SO}_{4}$ amount can influence the number of NAT particles nucleated. However, given the uncertainties in these parameters in this observation, it is not possible to conclusively state that this mechanism governed the NAT formation and produced the different number densities compared with Carslaw et al. [1998b].

4.3.3. Liquid particles. As explained in section 3.2, there is also a region within the cloud system of Plate 2 which shows backscatter properties of liquid aerosols only. Common to all air parcel trajectories that lead into this region is the short time within the ice cloud $(\approx 5 \mathrm{~min})$. Solid type I particles appear to be formed only in air parcels which spend about $15 \mathrm{~min}$ in the ice cloud. This is consistent with the observation that the small aerosol tail following the ice cloud measured at 1900 UTC (Plate 2d) shows no depolarization (data not shown here). So it seems that even if ice is formed, the nucleation of solids takes longer than $10 \mathrm{~min}$ (at least for the larger particles visible to the lidar).

\section{Summary}

We presented airborne lidar measurements of mountainwave-induced polar stratospheric clouds guided by forecasts of a mesoscale dynamical model. Ice clouds (PSC type II) were detected in the coldest regions predicted by the MM5 model directly above the mountain range and $300 \mathrm{~km}$ downstream. The dynamical simulations therefore appear to provide reliable estimations for the spatial and temporal extent of mesoscale temperature fluctuations. The model resolution proved not to be sufficient to reproduce the fine scale waves apparent in the lidar data ( $\approx 12 \mathrm{~km}$ wavelength). The resulting underestimation of the minimum temperatures was found to be about 3-4 K. It is concluded that the MM5-model calculations can serve to "renormalize" the results of simpler linearized dynamical models that tend to overestimate the mesoscale temperature fluctuations [Bacmeister et al., 1994] but which are computationally efficient enough to carry out 
global analyses of temperature anomalies induced by mountain waves.

The quasi-Lagrangian lidar measurements enabled us to carry out a detailed microphysical simulation. We found nearly full activation of the available liquid aerosol droplets to ice particles. To start this process, a super cooling of $4 \mathrm{~K}$ with respect to ice is needed. This is in line with the result of our first study of this kind [Carslaw et al., 1998b], although in the earlier case the cooling rates reached only half the values and large ternary solution droplets were present at the inflow side of the ice cloud. Therefore this relatively high supercooling seems to be a general feature of ice cloud formation in mountain wave PSCs.

In contrast to measurements of January 1995 here we found direct formation of solid hydrate particles within the ice cloud for a large number of nuclei but only in parts of the cloud where the ice phase lasted at least 10-15 min.

Different microphysical model runs showed that there is no significant dependence of the optical parameters measured by the lidar on the type of hydrate formed (NAD or NAT). Such a distinction seems to be not possible by optical remote sensing in the visible (where no strong wavelength dependence of the refractive index is present).

Acknowledgments. This study used data and software from ECMWF, the Finnish Mctcorological Institute (FMI) at Sodankylä and the National Center of Atmospheric Research (NCAR). The assistance by R. Kivi (FMI) and Y.Kuo (NCAR) is gratefully acknowledged. Part of this work was funded by the Bundesministerium für Bildung, Wissenschaft, Forschung und Technologie under contracts 07DLR01 and 01LO9506.

\section{References}

Appenzeller, C., and H.C. Davies, Structure of stratospheric intrusions into the troposphere, Nature, 358, 570-572, 1992.

Bacmeister, J.T., P.A. Newman, B.L. Gary, and K.R. Chan, An algorithm for forecasting mountain wave-related turbulence in the stratosphere, Weather Forecasting, 9, 241-253, 1994.

Baines, P.G., Topographic Effects in Stratified Flows, 482 pp., Cambridge Univ. Press, New York, 1995.

Barnes, J.E., and D.J. Hofmann, Lidar measurements of stratospheric aerosol over Mauna Loa Observatory, Geophys. Res. Lett., 24, 1923-1926, 1997.

Carslaw, K.S., et al., Increased stratospheric ozone depletion due to mountain-induced atmospheric waves, Nature, 391, 675-678, 1998a.

Carslaw, K.S., M. Wirth, A. Tsias, B. P. Luo, A. Dörnbrack, M. Leutbecher, H. Volkert, W. Renger, J. T. Bacmeister, and T. Peter, Particle microphysics and chemistry in remotely observed mountain polar stratospheric clouds, J. Geophys. Res., 103, 57855796, 1998 b.

Dörnbrack, A., M. Leutbecher, H. Volkert, and M. Wirth, Mesoscale forecasts of stratospheric mountain waves, Meteorol. Appl., 5, 117-126, 1998.

Dudhia, J., A non-hydrostatic version of the Penn State-NCAR Mesoscale Model: Validation tests and simulation of an Atlantic cyclone and cold front, Mon. Weather Rev., 121, 1493-1513, 1993.

Grell, G. A., J. Dudhia, and D. R. Stauffer, A description of the fifth-generation Penn State/NCAR mesoscale model (MM5), Techn. Note 398, 121 pp., Nat. Cent. for Atmos. Res., Boulder, Colo., 1994.

Hanson, D.R., and K. Mauersberger, Laboratory studies of the nitric acid trihydrate: Implications for the south polar stratosphere, Geophys. Res. Lett., 15, 855-858, 1988.
Keyser, L. F., and M. T. Leu, Morphology of Nitric Acid and Water Ice Films, Micros. Res. Technol., 25, 434-438, 1993.

Koop, T., K.S. Carslaw, and T. Peter, Thermodynamic stability and phase transitions of PSC particles, Geophys. Res. Lett., 24, 2199 2202, 1997.

Krieger, U., B.P. Luo, J. Mössinger, U. Weers, and T. Peter, Refractive indicies of $\mathrm{H}_{2} \mathrm{SO}_{4} / \mathrm{HNO}_{3} / \mathrm{H}_{2} \mathrm{O}$ solutions to stratospheric temperatures, paper presented at the 1997 Conference on the Atmospheric Effects of Aviation, NASA, Virginia Beach, Va., 1997.

Leutbecher, M., and H. Volkert, Stratospheric temperature anomalies and mountain waves: A three-dimensional simulation using a multi-scale weather prediction model, Geophys. Res. Lett., 23, 3329-3332, 1996.

Meilinger, S.K., T. Koop, B.P. Luo, T. Huthwelker, K.S. Carslaw, P.J. Crutzen, and T. Peter, Size-dependent stratospheric droplet composition in lee wave temperature fluctuations and their potential role in PSC freezing, Geophys. Res. Lett., 22, 3031-3034, 1995.

Mishchenko, M. I., Light scattering by randomly oriented axially symmetric particles, J. Opt. Soc. Am. 8, 871-882, 1991.

Pawson, S., B. Naujokat, and K. Labitzke, On the polar stratospheric cloud formation potential of the northern stratosphere, J. Geophys. Res., 100, 23,215-23,225,1995.

Peter, T., Microphysics and chemistry of polar stratospheric cloud particles, Ann. Rev. Phys. Chem., 48, 785-822, 1997.

Pierrehumbert, R.T., and B. Wyman, Upstream effects of mesoscale mountains, J. Atmos. Sci., 42, 977-1003, 1985.

Queney, P., The problem of air flow over mountains: A summary of theoretical studies, Bull. Am. Meteorol. Soc., 29, 16-27, 1948.

Shutts, G.J., M. Kitchen, and P.H. Hoare, A large amplitude gravity wave in the stratosphere detected by radiosonde, Q.J. R. Meteorol. Soc., 114, 579-694, 1988.

Störmer, C., Remarkable clouds at high altitudes, Nature, 123, 940941, 1929.

Tolbert, M.A., Stratospheric aerosols and polar stratospheric cloud formation, Science, 264, 527, 1994.

Tsias, A., A. Prenni, K.S. Carslaw, T. P. Onasch, B. P. Luo, M.A. Tolbert, and T. Peter, Freezing of polar stratospheric clouds in orographically-induced strong warming events, Geophys. Res. Lett., 24, 2303-2306, 1997.

Wirth, M., and W. Renger, Evidence of large scale ozone depletion within the arctic polar vortex 94/95 based on airborne LIDAR measurements, Geophys. Res. Lett., 23, 813-816, 1996.

World Meteorological Organization (WMO), Scientific Assessment of Ozone Depletion: 1991, WMO Report 25, Geneva, 1992.

World Meteorological Organization (WMO), Scientific Assessment of Ozone Depletion: 1994, WMO Report 37, Geneva, 1994.

Worsnop, D. R., L. E. Fox, M. S. Zahniser, and S. C. Wofsy, Vapor pressure of solid hydrates of nitric acid: Implications for PSCs, Science, 259, 71-74, 1993.

K. S. Carslaw, Environment Centre, University of Leeds, Leeds LS2 9JT, United Kingdom. (carslaw@lec.leeds.ac.uk)

A. Dörnbrack, M. Leutbecher, and H. Volkert, Institut für Physik der Atmosphäre, DLR-Oberpfaffenhofen, Postfach 1116, 82334 Wessling, Germany. (Andreas.Doernbrack@dlr.de; Martin.Leutbecher@dlr.de; Hans.Volkert@dlr.de)

T. Peter, Institute of Atmospheric Sciences, Eidgenössische Technische Hochschule, Hoenggerberg HPP, CH-8093 Zuerich, Switzerland. (peter@nike.mpch-mainz.mpg.de)

W. Renger, V. Weiß, and M. Wirth, Arbeitsgruppe Lidar, DLR-Oberpfaffenhofen, Postfach 1116, 82334 Wessling, Germany. (Wolfgang.Renger@dlr.de; Volker.Weiss@dlr.de; Martin.Wirth@dlr.de)

A. Tsias, Max-Planck-Institut für Chemie, Postfach 3060, Mainz. 55020, Germany. (tsias@mpch-mainz.mpg.de)

(Received May 20, 1998; revised December 1, 1998; accepted December 4, 1998.) 